Pacific Journal of Mathematics

ALMOST-PERIODIC FUNCTIONS WITH UNBOUNDED 


\title{
ALMOST-PERIODIC FUNCTIONS WITH UNBOUNDED INTEGRAL
}

\author{
RUSSELL A. JOHNSON
}

Let $B$ be an almost-periodic (a.p.) function with mean value zero. Let $G(t)=\int_{0}^{t} B(s) d s$. The well-known theorem of Bohr states that $G(t)$ is uniformly bounded iff $G(t)$ is a.p. This theorem may be reformulated in the following way. Let $\Omega$ be the hull of $B$, and let $(\Omega, R)$ be the flow on $\Omega$ defined by translation. Since $B$ is a.p., $\Omega$ is a compact abelian topological group. There is a continuous $b: \Omega \rightarrow R$ and an $\omega_{0} \in \Omega$ such that $b\left(\omega_{0} \cdot t\right)=B(t)$. I.e., $b$ "extends $B$ to $\Omega$ '. Then Bohr's theorem is equivalent to the following: $G(t)$ is bounded iff there is a continuous $r: \Omega \rightarrow R$ such that $r(\omega \cdot t)-r(\omega)=\int_{0}^{t} b(\omega \cdot s) d s(\omega \in \Omega, t \in \boldsymbol{R})$.

In this paper, we consider the case when $G(t)$ is unbounded. Two results are obtained. The first is a generalization of Bohr's theorem: let $\mu$ be (normalized) Haar measure on $\Omega$, and let $g_{\omega}(t)=\int_{0}^{t} b(\omega \cdot s) d s(\omega \in \Omega, t \in R)$; then $\varlimsup_{n \rightarrow \infty} 1 / 2 n \gamma\left\{t \in[-n, n] \mid g_{\omega}(t) \in I\right\}>0$ for some compact $I \subset R$ and some $\omega \in \Omega$ iff there exists a $\mu$-measurable $r: \Omega \rightarrow R$ such that $r(\omega \cdot t)-r(\omega)=\int_{0}^{t} b(\omega \cdot s) d s(\omega \in \Omega, t \in R)$. Here $\gamma$ is Lebesgue measure on $R$. Thus, $r$ exists if some $g_{\omega}(t)$ is not too badly unbounded. This theorem is stated for the class of "minimal" functions (see below), which includes the a.p. ones.

Now, an example in ([10]) shows that there exist a.p. functions $b$ iwith $g_{w}(t)$ unbounded which admit a discontinuous, $\mu$-measurable $r$ as above. It is natural to ask whether $r$ always exists. Our second result $(\S 4)$ states that this is false; residually many functions $b \in C(\Omega)$ with mean value zero admit no $\mu$-measurable $r$. This is, at first glance, a bit disappointing. However, combining our two theorems, we can at least draw this conclusion: even a "measure-theoretic" Bohr's theorem applies to only a small (though non-vacuous) set of a.p. functions.

The proof of the first result may be of interest. We make use of techniques and 'results from ergodic theory, lifting theory ([9]), and the theory of linear skew-product flows ([14], [15]). Of special importance is a close examination of a disintegration ([3], [9]) of a certain ergodic measure. Said examination involves a deep theorem of Furstenberg concerning such disintegrations ([7], Theorem 4.1). 
His theorem is stated for integer flows. Since our interest is in real flows, we extend his theorem to this case (in fact, to the case of an arbitrary phase group; we also make other generalizations. See $2.2,2.3,2.4)$. The extension is performed by mimicking Furstenberg's proof.

1. Preliminaries. In $1.1-1.7, X$ is a locally compact Hausdorff space unless there is a statement to the contrary.

Definitions 1.1. Let $M(X)$ be the set of nonnegative (Radon) measures on $X$ ([1], Chpt. III, $\S 1, \mathrm{n}^{\circ} 3$, Def. 2 ). We will always give $M(X)$ the topology of pointwise convergence (i.e., $\mu_{n} \rightarrow \mu$ iff $\mu_{n}(f) \rightarrow \mu(f)$ for each continuous $f: X \rightarrow C$ with compact support). Let $M_{1}(X)=\{\mu \in M(X) \mid\|\mu\| \equiv \mu(X)=1\}$. If $\mu \in M(X)$, we use

$$
\int_{X} f(x) d \mu(x), \quad \int_{X} f d \mu,
$$

or $\mu(f)$ to denote an integral with respect to $\mu$. Let Supp $\mu$ be the support of $\mu$.

Definitions, Remarks 1.2. Let $\mu \in M(X)$, and let $\pi$ map $X$ to a topological space $Y$. Say $\pi$ is $\mu$-Lusin-measurable if, for each compact $K \subset X$ and $\varepsilon>0$, there is a compact $K_{1} \subset K$ such that $\mu\left(K \sim K_{1}\right)<\varepsilon$ and $\pi \mid K_{1}$ is continuous. If $Y$ is separable metric, then $\pi$ is $\mu$-Lusin-measurable iff $\pi^{-1}(B)$ is $\mu$-measurable for every clised ball $B \subset Y$. See ([1], Chpt. IV, $\S 5$, Prop. 1 of $n^{\circ} 1$ and Thm. 4 of $\left.n^{\circ} 5\right)$.

Definitions, Remarks 1.3. Let $Y$ be locally compact Hausdorff, let $\mu \in M(X)$, and let $\pi: X \rightarrow Y$ be $\mu$-Lusin-measurable. Say $\pi$ is $\mu$ proper if, for every compact $C \subset Y, \pi^{-1}(C)$ is essentially $\mu$-integrable (i.e., $\sup _{K} \mu\left(\pi^{-1}(C) \cap K\right)<\infty$, where $K \subset X$ is compact). If $\pi$ is $\mu$ proper, one can define an image measure $\nu=\pi(\mu)$ ([2], $\S 6, \mathrm{n}^{\circ} 1$, Def. 1). If $X$ is compact and $\pi$ is $\mu$-Lusin-measurable, then $\pi$ is necessarily $\mu$-proper. If $X$ and $Y$ are compact, then $\nu=\pi(\mu)$ has the following property: $f \in L^{1}(Y, \nu)$ iff $f \circ \pi \in L^{1}(X, \mu)$, and

$$
\int_{Y} f d \nu=\int_{X} f \circ \pi d \mu
$$

See $\left([2], \S 6, \mathrm{n}^{\circ} 2\right.$, Thm. 1).

Definition 1.4. Let $X$ and $Y$ be compact Hausdorff, $\mu \in M(X)$, $\pi: X \rightarrow Y$ a $\mu$-proper map, and $\nu=\pi(\mu) . A$ map $\lambda: Y \rightarrow M(X): y \rightarrow \lambda_{y}$ is a disintegration of $\mu$ with respect to $\nu$ (or with respect to $\pi$ ) if: 
(a) $\operatorname{Supp} \lambda_{y} \subset \pi^{-1}(y)(y \in Y)$;

(b) $\left\|\lambda_{y}\right\|=1(y \in Y)$;

(c) $\lambda$ is $\nu$-adequate ([2], $\S 3, \mathrm{n}^{\circ} 1$, Def. 1$)$; see also $1.5(\mathrm{a})$;

(d) if $f: X \rightarrow \boldsymbol{R}$ is continuous, then $y \rightarrow \lambda_{y}(f)$ is $\nu$-integrable, and $\int_{X} f(x) d \mu(x)=\int_{Y} \lambda_{y}(f) d \nu(y)$.

REMARKS 1.5. (a) If $\lambda$ is $\nu$-Lusin-measurable, it is $\nu$-adequate ([2], $\S 3, n^{\circ} 1$, Prop. 2).

(b) One can define the notion of disintegration if $X$ and $\mathrm{Y}$ are locally compact; slight modifications are needed in 1.4(d). See [3], $\S 3, \mathrm{n}^{\circ} 1$, Thm. 1).

TheOREM 1.6. Let $X$ and $Y$ be compact metric, $\pi: X \rightarrow Y$ a $\mu$ proper map, $\nu=\pi(\mu)$.

(a) There exists a disintegration $\lambda$ of $\mu$ with respect to $\nu$.

(b) If $\lambda^{\prime}: Y \rightarrow M(X)$ is another map satisfying (a), (c), and (d) of 1.4, then $\lambda^{\prime}=\lambda \nu$ - a.e.

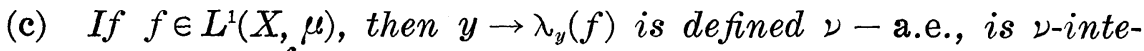
grable, and $\mu(f)=\int_{Y} \lambda_{y}(f) d \nu(y)$.

Parts (a) and (b) of 1.6 follow from a more general theorem, in which $X$ and $Y$ are locally compact second countable ([3], $\S 3, \mathrm{n}^{\circ} 1$, Thm. 1). Part 1.6(c) follows from 1.6(a) and ([2], $\S 3, \mathrm{n}^{\circ} 3$, Thm. 1).

Definition 1.7. Let $\mu \in M(X)$, and let $M^{\infty}(X, \mu)=\{f: X \rightarrow \boldsymbol{R} \mid f$ is bounded and $\mu$-measurable $\} . \mathrm{A} \operatorname{map} \rho: M^{\infty}(X, \mu) \rightarrow M^{\infty}(X, \mu)$ is a lifting of $M^{\infty}(X, \mu)$ if (i) it is linear, (ii) $\rho(f)=f$ locally $\mu$-a.e.; (iii) if $f_{1}=f_{2}$ locally $\mu$-a.e., then $\rho\left(f_{1}\right)=\rho\left(f_{2}\right)$ everywhere; (iv) $f \geqq$ $0 \Rightarrow \rho(f) \geqq 0$; (v) $\rho\left(f_{1} \cdot f_{2}\right)=\rho\left(f_{1}\right) \cdot \rho\left(f_{2}\right)$. If, in addition, (vi) $\rho(f)=f$ for every continuous $f \in M^{\infty}(X, \mu)$, then $\rho$ is a strong lifting of $M^{\infty}(X, \mu)$. See ([9], Chpt. III, Def. 1).

THEOREM 1.8 ([8]). Let $X$ be a locally compact topological group with left Haar measure $\mu$. There exists a strong lifting $\rho$ of $M^{\infty}(X, \mu)$ which commutes with left translations (thus, let $\left(T_{x} f\right)(\bar{x})=$ $f(x \cdot \bar{x})\left(f \in M^{\infty}(X, \mu) ; x, \bar{x} \in X\right)$; one has $\left.\rho\left(T_{x} f\right)=T_{x}(\rho(f))\right)$.

Definitions 1.9. A (right) transformation group (or flow) is a triple $(X, T, \Phi)$, where $X$ is a topological space, $T$ is a topological group, and $\Phi: X \times T \rightarrow X:(x, t) \rightarrow x \cdot t$ is a continuous map such that: (i) $x \cdot \mathrm{idy}=x(x \in X$; idy $=$ identity in $T)$; (ii) $(x \cdot t) \cdot s=x \cdot(t \cdot s)(x \in X$; $t, s \in T)$. We will always suppress $\Phi$, writing just $(X, T)$ when 
referring to a flow. If $t \in T$ and $A \subset X$, define $A \cdot t=\{x \cdot t \mid x \in A\}$. If $t \in T$ and $f: X \rightarrow Y$, define $(t \cdot f)(x)=f(x \cdot t)(x \in X)$. If $X$ is compact Hausdorff and $\mu \in M(X)$, define $(\mu \cdot t)(f)=\mu(t \cdot f)(t \in T, f \in C(X))$. Equivalently, one could define $(\mu \cdot t)(A)=\mu\left(A \cdot t^{-1}\right)$ for each $\mu$-measurable $A \subset X$.

Definitions, Remarks 1.10. Let $(X, T)$ be a flow with $X$ compact Hausdorff. Let $\mu \in M_{1}(X)$. A set $A \subset X$ is T-invariant if $\mu(A$. $t \Delta A)=0$ for each $t \in T$. It is strictly $T$-invariant if $A \cdot t=A(t \in T)$. The element $\mu$ of $M_{1}(X)$ is T-invariant if $\mu \cdot t=\mu(t \in T)$. It is $T$ ergodic if, in addition, $\mu(A)=0$ or $\mu(A)=1$ for every $T$-invariant set $A$. If $(X, T)$ has only one invariant measure $\mu$, then $\mu$ is ergodic ([13]).

Definitions 1.11. Let $B: \boldsymbol{R} \rightarrow \boldsymbol{R}^{n}$ be a uniformly bounded, uniformly continuous map. Let $C\left(\boldsymbol{R}, \boldsymbol{R}^{n}\right)$ be the space of continuous maps from $\boldsymbol{R}$ to $\boldsymbol{R}^{n}$, with the compact-open topology. For each $\tau \in R$, define $f_{z}(t)=f(t+\tau)\left(f \in C\left(\boldsymbol{R}, \boldsymbol{R}^{n}\right), t \in \boldsymbol{R}\right)$, and let $\Omega=\operatorname{cls}\left\{B_{z} \mid \tau \in\right.$ $\boldsymbol{R}\} \subset C\left(\boldsymbol{R}, \boldsymbol{R}^{n}\right)$. Then $\Omega$ is compact metric ([12]), and the translation $(f, \tau) \rightarrow f_{\tau}$ induces a flow $(\Omega, \boldsymbol{R})$. The space $\Omega$ is the hull of $B$. Let $\omega_{0}$ represent the element $B$ of $\Omega$. Define $b: \Omega \rightarrow \boldsymbol{R}^{n}: b(\omega)=\omega(0)$. Then $b\left(\omega_{0} \cdot t\right)=B_{t}(0)=B(t)$. Thus $b$ "extends $B$ to $\Omega$ ". If $B(t)$ is almost periodic, then ([5]) $\Omega$ is a compact abelian topological group, with dense subgroup $\boldsymbol{R}$; the flow $(\Omega, \boldsymbol{R})$ is defined by the group operation $(\omega \cdot t$ is the product of $\omega \in \Omega$ and $t \in R \subset \Omega)$. The unique invariant measure for $(\Omega, \boldsymbol{R})$ is normalized Haar measure. If $(\Omega, \boldsymbol{R})$ is minimal (i.e., the only nonempty closed invariant subset of $\Omega$ is $\Omega$ itself), we say that $B$ is minimal. If $B$ is a.p., then $B$ is minimal.

2. Furstenberg's theorem. In this section, we generalize Furstenberg's theorem. We have tried to compromise between, on the one hand, ignoring the fact that Furstenberg's proof is readily available, and, on the other, giving no details at all and simply giving references to that proof.

Notation 2.1. For the most part, we adopt the notation of ([7]). However, a disintegration of a measure $\mu$ will be written $\omega \rightarrow \mu_{\omega}$, rather than $\omega \rightarrow \mu(\omega)$. Compare also with 1.4 , where we let $\lambda$ denote a disintegration. If $B$ is a set, we let $|B|$ be its cardinality. Let $\Omega$ denote a compact metric space. If $(\Omega, T)$ is a flow, we sometimes write $\omega t$ for $\omega \cdot t(\omega \in \Omega, t \in T)$. In $\S 2, T$ is an arbitrary topological group. 
THEOREM 2.2. Let $(\Omega, T)$ be a flow with $\mu_{0}$ a T-ergodic measure on $\Omega$. Let $K$ be the unit circle, let $\Sigma=\Omega \times K$, and let $\pi: \Sigma \rightarrow \Omega$ : $(\omega, \zeta) \rightarrow \omega$ be the projection. Let $(\Sigma, T)$ be a flow satisfying $\pi((\omega$, $\zeta) \cdot t)=[\pi(\omega, \zeta)] \cdot t=\omega \cdot t((\omega, \zeta) \in \Sigma) . \quad($ Equivalently, suppose $(\omega, \zeta) \cdot t=$ $\left(\omega t, h_{t}(\omega, \zeta)\right)$ for continuous functions $\left.h_{t}: \Sigma \rightarrow K\right)$. Let $\mu$ be $a$ measure ergodic with respect to $(\Sigma, T)$ such that $\pi(\mu)=\mu_{0}$, and let $\omega \rightarrow \mu_{\omega}: \Omega \rightarrow M_{1}(\Sigma)$ be a disintegration of $\mu$ with respect to $\mu_{0}(1.6)$. If $\mu$ is not the only ergodic measure on $(\Sigma, T)$ such that $\pi(\mu)=\mu_{0}$, then there is an integer $n$ such that $\left|\operatorname{Supp} \mu_{\omega}\right|=n \mu_{0}$ - a.e.

Proof. We divide the proof into steps.

(1) Let $t \in T$, and define $\tilde{\mu}_{\omega} \in M_{1}(\Sigma)$ by $\tilde{\mu}_{\omega}(f)=\mu_{\omega t}\left(t^{-1} f\right)(f \in C(\Sigma))$. Clearly $\omega \rightarrow \bar{\mu}_{\omega}$ satisfies $1.4(\mathrm{a}),(\mathrm{b}),(\mathrm{c})$. Since

$$
\begin{aligned}
\int_{\Omega} \tilde{\mu}_{\omega}(f) d \mu_{0}(\omega)=\int_{\Omega} \mu_{\omega t}\left(t^{-1} f\right) d \mu_{0}(\omega) & =(\text { by } 1.3) \\
\int_{\Omega} \mu_{\omega}\left(t^{-1} f\right) d \mu_{0}(\omega)=\mu\left(t^{-1} f\right) & =\mu(f), \quad \omega \longrightarrow \tilde{\mu}_{\omega}
\end{aligned}
$$

satisfies $1.4(\mathrm{~d})$. By 1.6(b), $\tilde{\mu}_{\omega}=\mu_{\omega} \nu$-a.e.; i.e., $=\left(\mu_{\omega}\right) \cdot t \mu_{0}$-a.e. for each fixed $t$.

(2) For each integer $n$, let $B_{n}=\left\{\omega \in \Omega|| \operatorname{Supp} \mu_{\omega} \mid \leqq n\right\}$. We claim $B_{n}$ is $\mu_{0}$-measurable. For, let $\Gamma$ be a compact set such that $\omega \rightarrow \mu_{\omega}$ is continuous on $\Gamma$. It suffices to show that $B \cap \Gamma$ is closed. Let $\omega_{l} \rightarrow \omega, \omega_{l} \in B_{n} \cap \Gamma$. Suppose $\mu_{\omega_{l}}$ is supported on points

$$
\left(\omega_{l}, \zeta_{1}\left(\omega_{l}\right)\right), \cdots,\left(\omega_{l}, \zeta_{k}\left(\omega_{l}\right)\right)(k \leqq n) ;
$$

letting $\delta_{\rho}$ denote the Dirac measure at $\rho$, we write

$$
\mu_{\omega_{l}}=\sum_{i=1}^{n} \alpha_{i}\left(\omega_{l}\right) \delta_{\left(\omega_{l}, \zeta_{i}\left(\omega_{l}\right)\right)},
$$

where $0=\alpha_{k+1}\left(\omega_{l}\right)=\cdots=\alpha_{n}\left(\omega_{l}\right)$ if $k<n$. Choosing a subsequence, we assume $\alpha_{i}\left(\omega_{l}\right) \rightarrow \bar{\alpha}_{i}, \zeta_{i}\left(\omega_{l}\right) \rightarrow \bar{\zeta}_{i}(1 \leqq i \leqq n)$. If $f \in C(\Sigma)$, then $\mu_{\omega}(f)=$ $\lim _{l \rightarrow \infty} \mu_{\omega_{l}}(f)=\sum_{i=1}^{n} \bar{\alpha}_{i} f\left(\omega, \bar{\zeta}_{i}\right)$.

(a) Suppose $\Delta=\operatorname{Supp} \mu_{\omega}$ is infinite. Since $K$ is compact, $\mu_{\omega}$ assigns positive measure to open subsets of $\Delta$. Let $V$ be an open set in $\Delta$ whose closure does not contain $\bar{\zeta}_{1}, \cdots, \bar{\zeta}_{n}$. Let $0 \leqq f \in C(\Sigma)$ be equal to 1 on $V$, and equal to zero at $\left(\omega, \bar{\zeta}_{i}\right)(1 \leqq i \leqq n)$. We obtain a contradiction; hence $\mid$ Supp $\mu_{\omega} \mid<\infty$.

(b) Suppose $\left|\operatorname{Supp} \mu_{\omega}\right|<\infty$, with $\mu_{\omega}=\sum_{i=1}^{r} \alpha_{i} \delta_{\left(\omega, \zeta_{i}\right)}$. Let $\bar{\alpha}_{i}, \bar{\zeta}_{i}$ be as above. Then

(*) each $\zeta_{i} \in\left\{\bar{\zeta}_{j} \mid \bar{\alpha}_{j} \neq 0,1 \leqq j \leqq n\right\}$, if $\alpha_{i} \neq 0$;

$\left(^{* *}\right)$ each $\bar{\zeta}_{i} \in\left\{\zeta_{j} \mid \alpha_{j} \neq 0,1 \leqq j \leqq r\right\}$, if $\bar{\alpha}_{i} \neq 0$. For suppose $(*)$ is false. Choose $0 \leqq f \in C(\Sigma)$ such that $f\left(\zeta_{i}\right)=1$ and $f\left(\bar{\zeta}_{j}\right)=0$ if 
$\bar{\alpha}_{j} \neq 0$; one obtains a contradiction. Similarly for $\left(^{* *}\right)$. Now, by $\left({ }^{*}\right)$, we must have $\left|\operatorname{Supp} \mu_{\omega}\right| \leqq n$; hence $\mu_{\omega} \in B_{n}$.

We have shown $B_{n}$ is $\mu_{0}$-measurable. As a corollary to the proof, $C_{n}=\left\{\omega \in \Omega|| \operatorname{Supp} \mu_{\omega} \mid>n\right\}$ is $\mu_{0}$-measurable, since $C_{n} \cap \Gamma$ is open in $\Gamma$.

From (1) and (2), we obtain two conclusions.

(3) If $D=\left\{\omega \in \Omega|| \operatorname{Supp} \mu_{\omega} \mid<\infty\right\}$, then $D=\bigcup_{n=1}^{\infty} B_{n}$ is $\mu_{0}$ measurable. Also, $D$ is $T$-invariant; hence $\mu_{0}(D)=0$ or 1 .

(4) Note $D=\bigcup_{n=1}^{\infty} D_{n}$, where $D_{n}=B_{n} \cap C_{n-1}$ is $\mu_{0}$-measurable and $T$-invariant. Suppose $\nu(D)=1$. Then, for some $n, D_{n}=\{\omega \in$ $\left.\Omega|| \operatorname{Supp} \mu_{\omega} \mid=n\right\}$ has $\mu_{0}$-measure 1 .

From now through (15), assume for contradiction that $\left|\operatorname{Supp} \mu_{\omega}\right|=$ $\infty \mu_{0}$ - a.e. Let $\mu^{\prime}$ be another ergodic measure on $\Sigma$, with disintegration $\omega \rightarrow \mu_{\omega}^{\prime}$, such that $\pi\left(\mu^{\prime}\right)=\mu_{0}$.

(5) As on p. 593 of ([7]), one can show that $\mu_{\omega}$ is nonatomic (i.e., no point has nonzero measure) $\mu_{0}$-a.e. As on p. 594 of ([7]), one can show that $\mu_{\omega}^{\prime}$ is also nonatomic $\mu_{0}$ - a.e.

Let $\lambda=1 / 2\left(\mu+\mu^{\prime}\right)$. Then $\omega \rightarrow \lambda_{\omega}=1 / 2\left(\mu_{\omega}+\mu_{\omega}^{\prime}\right)$ is a disintegration of $\lambda$ with respect to $\mu_{0}$, and $\lambda_{\omega}$ is $\mu_{0}$-a.e. nonatomic. Fix $\zeta_{0} \in$ $K$, let $\left\{\zeta_{0}, \zeta\right\} \subset K$ denote the interval from $\zeta_{0}$ to $\zeta$ (counterclockwise), and let $K^{\prime}$ be the unit circle in the complex plane. Let $\pi^{\prime}: \Omega \times K^{\prime} \rightarrow$ $\Omega$ denote the projection $(\omega, \zeta) \rightarrow \omega$.

Define

$$
\psi: \Sigma \longrightarrow \Omega \times K^{\prime}:(\omega, \zeta) \longrightarrow\left(\omega, e^{2 \pi i \lambda_{\omega}\left(1 \zeta_{0}, \zeta[)\right.}\right),
$$

and for each $t$,

$$
g_{t}: \Omega \longrightarrow K^{\prime}: \omega \longrightarrow e^{2 \pi i \lambda_{\omega t}\left(\left(\xi_{0}, h_{t}\left(\omega, \xi_{0}\right) !\right)\right.} .
$$

For each $t \in T$, define $\tilde{t}: \Omega \times K^{\prime} \rightarrow \Omega \times K^{\prime}:(\omega, \zeta) \rightarrow\left(\omega \cdot t, g_{t}(\omega) \zeta\right)$. Denote the image of $(\omega, \zeta)$ under $\widetilde{t}$ by $(\omega, \zeta) \cdot \widetilde{t}$. As on p. 594 of ([7]), one has

(8) $\psi((\omega, \zeta) \cdot t)=[\psi(\omega, \zeta)] \cdot \widetilde{t}$ for $(\omega, \zeta) \in \pi^{-1}(B)$, where $B \subset \Omega$ has $\mu_{0}$-measure 1 ( $B$ depends on $t$ ).

(9) We show that $\psi$ is $\eta$-Lusin-measurable for any measure $\eta$ on $\Sigma$ such that $\pi(\eta)=\mu_{0}{ }^{1}$ (in particular, for $\mu, \mu^{\prime}, \lambda$ ). If $\zeta_{0} \neq \zeta \in K$ and $m>1$, construct continuous functions $\widetilde{g}_{m, \zeta}: K \rightarrow \boldsymbol{R}$ such that (i) $\lim _{m \rightarrow \infty} \widetilde{g}_{m, \zeta}(\bar{\zeta})=\varphi_{\left\{\zeta_{0}, \zeta\right\}}(\bar{\zeta})$ (here $\varphi$ denotes characteristic function); (ii) for fixed $m, \widetilde{g}_{m, \zeta_{n}} \rightarrow \widetilde{g}_{m, \zeta}$ uniformly if $\zeta_{n} \rightarrow \zeta$; (iii) $0 \leqq \widetilde{g}_{m, \zeta}\left(\zeta^{\prime}\right) \leqq 1$ for all $m, \zeta, \bar{\zeta}$. Let $g_{m, \zeta}(\omega, \bar{\zeta}) \equiv \widetilde{g}_{m, \zeta}(\bar{\zeta})$. Define $\tau_{m}: \Sigma \rightarrow \boldsymbol{R}:(\omega, \zeta) \rightarrow \lambda_{\omega}\left(g_{m, \zeta}\right)$. Let $\Gamma \subset \Omega$ be a compact set on which $\omega \rightarrow \lambda_{\omega}$ is continuous. Let $\left(\omega_{n}, \zeta_{n}\right) \rightarrow(\omega, \zeta)$ in $\pi^{-1}(\Gamma)$. Then $\left|\lambda_{\omega_{n}}\left(g_{m, \zeta_{n}}\right)-\lambda_{\omega}\left(g_{m, \zeta}\right)\right| \leqq \mid \lambda_{\omega_{n}}\left(g_{m, \zeta_{n}}\right)-$ $\lambda_{\omega_{n}}\left(g_{m, \zeta}\right)|+| \lambda_{\omega_{n}}\left(g_{m, \zeta}\right)-\lambda_{\omega}\left(g_{m, \zeta}\right)\left|\leqq \| g_{m, \zeta_{n}}-g_{m, \zeta}\right||+| \lambda_{\omega_{n}}\left(g_{m, \zeta}\right)-$

${ }^{1}$ Here and in (11) below, we assume $\eta$ admits a disintegration $\omega \rightarrow \eta_{\omega}$ with $\eta_{\omega}$ nonatomic for all $\omega$ (hence $\eta\left(\Omega \times\left\{\zeta_{0}\right\}\right)=0$ ). 
$\lambda_{\omega}\left(g_{m, \xi}\right) \mid \rightarrow 0$ as $n \rightarrow \infty$. Hence $\tau_{m}$ is $\eta$-Lusin-measurable. Now, $\lim _{m \rightarrow \infty} \tau_{m}(\omega, \zeta)=\lambda_{\omega}\left(\left\{\zeta_{0}, \zeta\right\}\right)$. Hence $\psi$ is $\eta$-Lusin-measurable.

(10) In a similar fashion, each $g_{t}$ is $\mu_{0}$-Lusin-measurable. Hence each map $\tilde{t}$ is $\eta^{\prime}$-Lusin-measurable for any measure $\eta^{\prime}$ on $\Omega \times K^{\prime}$ which satisfies $\pi^{\prime}\left(\eta^{\prime}\right)=\mu_{0}$.

(11) By 1.3 and (9), $\psi$ is $\eta$-proper if $\pi(\eta)=\mu_{0}$. By 1.8 and (10), $\tilde{t}$ is $\eta^{\prime}$-proper if $\pi^{\prime}\left(\eta^{\prime}\right)=\mu_{0}$. Hence $\psi(\mu) \equiv \mu_{*}$ and $\psi\left(\mu^{\prime}\right) \equiv \mu_{*}^{\prime}$ are Radon measures, and have unique disintegrations with respect to $\mu_{0}(1.6)$. As on p. 595 of $([7]), \psi(\lambda)=\mu_{0} \times m$, where $m$ is normalized Lebesgue measure on $K^{\prime}$. Moreover, by (8), (10), and ([2], $\S 6, \mathrm{n}^{\circ} 3$, Prop. $\left.4(\mathrm{a})\right)$, one has $\mu_{*} \cdot \tilde{t}=\psi(\mu \cdot t)=\psi(\mu)=\mu_{*}$. Similarly $\mu_{*}^{\prime} \cdot \tilde{t}=\mu_{*}^{\prime},\left(\mu_{0} \times m\right) \cdot \tilde{t}=\mu_{0} \times m(t \in T)$.

(12) Note $\mu_{0} \times m=1 / 2\left(\mu_{*}+\mu_{*}^{\prime}\right)$. We show that the assumption $\mu_{*} \neq \mu_{*}^{\prime}$ implies the existence of an $f \in L^{1}\left(\Omega \times K^{\prime}, \mu_{0} \times m\right)$ such that (i) for each $t, f((\omega, \zeta) \cdot \widetilde{t})=f(\omega, \zeta) \mu_{0} \times m$ - a.e.; (ii) $f$ is not equal to a constant $\mu_{0} \times m$-a.e. (The existence of $f$ does not follow from standard ergodic theory, since the flow $(\omega, \zeta, t) \rightarrow(\omega, \zeta) \cdot \tilde{t}$ has not been proved measurable. However, we need only imitate a standard proof.) Note that $\mu_{*} \ll \mu_{0} \times m$. Hence, if $E \subset \Omega \times K^{\prime}$ is $\mu_{*}$-measurable, then $\mu_{*}(E)=\int_{E} f d\left(\mu_{0} \times m\right)$ for a unique $f \in L^{1}\left(\mu_{0} \times\right.$ $m)$. Now, $\mu_{*}(E)=(1.3$ and $(11)) \mu_{*}\left(E \cdot \tilde{t}^{-1}\right)=\int_{E \cdot-1} f d\left(\mu_{0} \times m\right)=(1.3$ and $(11)) \int_{E} f((\omega, \zeta) \cdot \tilde{t}) d\left(\mu_{0} \times m\right)(\omega, \zeta)$. Hence $f\left((\omega, \zeta) \cdot \tilde{t}=f(\omega, \zeta) \mu_{0} \times\right.$ $m$ - a.e. for each $\tilde{t}$, and (i) is proved. If $f=$ const. $\mu_{0} \times m$-a.e., then const. $=1\left(\right.$ let $\left.E=\Omega \times K^{\prime}\right)$. But then $\mu_{*}=\mu_{0} \times m$, contradicting $\mu_{*} \neq \mu_{*}^{\prime}$. So (ii) holds, also.

(13) Using Fubini's theorem, expand $f$ in a partial Fourier series: $f \sim \sum_{m-\infty}^{\infty} a_{m}(\omega) \zeta^{m}$. Fix $t \in T$. By (i) in (12) and uniqueness of Fourier coefficients, $a_{m}(\omega \cdot t) g_{t}^{m}(\omega)=a_{m}(\omega) \mu_{0}$ - a.e. $(-\infty<m<\infty)$. Since $\mu_{0}$ is ergodic, $f$ is not a function of $\omega$ alone (otherwise (ii) of (12) is violated). Hence there exists $k \neq 0$ with $a_{k}(\omega) \neq 0$. Arguing as in Lemma 2.1 of ([7]), we see that, for each $t \in T, g_{t}^{k}(\omega)=R(\omega \cdot t) /$ $R(\omega) \mu_{0}$ - a.e., where $|R(\omega)|=1 \mu_{0}$-a.e. (in fact, $R(\omega)=\overline{a_{k}(\omega) \mid}$ $\left.\left|a_{k}(\omega)\right|\right)$.

(14) As on p. 595 of ([7]), let $J \subset K^{\prime}$ be any interval, and let $\Lambda^{\prime}(J)=\left\{(\omega, \zeta) \in \Omega \times K^{\prime} \mid R(\omega)^{-1} \zeta^{k} \in J\right\}$. Then (p. 595) $\mu_{0} \times m\left(\Lambda^{\prime}(J)\right)=$ $m(J)$. By (11) and (13), and arguing as on p. 595, one has $\mu_{0} \times$ $m\left(\Lambda^{\prime}(J) \cdot \widetilde{t} \Delta \Lambda^{\prime}(J)\right)=0$ for each $t \in T$. By (8) and (11), $\lambda(\Lambda(J) \cdot t \Delta \Lambda(J))=$ 0 if $\Lambda(J)=\psi^{-1}\left(\Lambda^{\prime}(J)\right)(t \in T)$. Also, $\lambda(\Lambda(J))=m(J)$. So $\lambda$ has invariant sets of all measures. Argue as on p. 595 again to obtain a contradiction to the assumption $\mu_{*} \neq \mu_{*}^{\prime}$. We conclude $\mu_{*}=\mu_{*}^{\prime}$.

(15) Note $\left.\psi\right|_{i \omega i \times K}$ is continuous for $\mu_{0}-$ a.a. $\omega$. Hence $\mu_{*, \omega} \equiv$ $\psi\left(\mu_{\omega}\right)$ and $\mu_{*, \omega}^{\prime} \equiv \psi\left(\mu_{\omega}^{\prime}\right)$ are defined $\mu_{0}-$ a.e., and can be shown to be disintegrations of $\mu_{*}, \mu_{*}^{\prime}$ with respect to $\mu_{0}$. By $1.6(\mathrm{~b}), \mu_{*, \omega}=$ 
$\mu_{0}$ - a.e. As on p. 595 of ([7]), it follows that $\mu_{\omega}=\mu_{\omega}^{\prime} \mu_{0}-$ a.e., and hence that $\mu=\mu^{\prime}$.

We have contradicted our assumption that $\left|\operatorname{Supp} \mu_{\omega}\right|=\infty \mu_{0}$-a.e. By (3), $\left|\operatorname{Supp} \mu_{\omega}\right|<\infty \mu_{0}$ - a.e., and by (4), $\left|\operatorname{Supp} \mu_{\omega}\right|=n \mu_{0}$ - a.e. for some $n$.

THEOREM 2.3. 1.2 remains true if $\Omega$ is compact Hausdorff.

Proof. The proof is not a repetition of that just given, since 1.6 does not now apply. Even if it did, a map $\omega \rightarrow \mu_{\omega}$ which satisfies 1.4(a), (b), (c), (d) need not be $\mu_{0}$ - Lusin measurable since $M_{1}(\Sigma)$ is not metrizable. (We used $\mu_{0}$ - Lusin-measurable of $\omega \rightarrow \mu_{\omega}$ heavily.) However, note that $K$ acts freely ([3]) on $\Omega \times K$ by group multiplication. By ([11], Theorem 1.9), every measure $\eta$ on $\Sigma(T$-invariant or not), has a $\pi(\eta)$-Lusin-measurable disintegration $\omega \rightarrow \eta_{\omega}$ with respect to $\pi(\eta)$; moreover, $\omega \rightarrow \eta_{\omega}$ is unique in the sense that, if $\omega \rightarrow \bar{\eta}_{\omega}$ is another $\pi(\eta)$-Lusin-measurable disintegration, then $\bar{\eta}_{\omega}=\eta_{\omega} \pi(\eta)-$ a.e.

Now go through the proof of 2.2, using $\mu_{0}$-Lusin-measurable disintegrations $\omega \rightarrow \mu_{\omega}, \omega \rightarrow \mu_{\omega}^{\prime}$. Nothing changes in steps (1)-(10), except that sequences are replaced by nets in various places. In (11), "unique disintegrations" is replaced by "unique $\mu_{0}$-Lusin-measurable disintegrations". All is the same in steps (12)-(14). In (15), however, we hit a snag. It is not clear that the maps $\omega \rightarrow \mu_{*, \omega}$ and $\omega \rightarrow \mu_{*, \omega}^{\prime}$ are $\mu_{0}$-Lusin-measurable; hence we cannot apply uniqueness to conclude that $\mu_{*, \omega}=\mu_{*, \omega}^{\prime} \mu_{0}$ - a.e. We escape as follows. Define $\mu_{*, \omega}=\psi\left(\mu_{\omega}\right), \mu_{*, \omega}^{\prime}=\psi\left(\mu_{w}^{\prime}\right)$. Recall $K^{\prime}$ is the unit circle. Let $\pi_{2}: \Omega \times K^{\prime} \rightarrow K^{\prime}$ be the projection. Define elements $\alpha_{\omega}, \alpha_{w}^{\prime} \in M_{1}\left(K^{\prime}\right)$ by $\alpha_{\omega}(h)=\mu_{*, \omega}\left(h \circ \pi_{2}\right), \alpha_{\omega}^{\prime}(h)=\mu_{*, \omega}^{\prime}\left(h \circ \pi_{2}\right)$. Let $f \in L^{1}\left(\Omega, \mu_{0}\right)$. Note

$$
\begin{aligned}
\int_{\varrho \times K^{\prime}} & f(\omega) h \circ \pi_{2}(\omega, \zeta) d \mu_{*}(\omega, \zeta)=(1.3) \int_{\Sigma}\left(f \cdot h \circ \pi_{2}\right) \circ \psi d \mu=(1.6 \mathbf{c}) \\
& \int_{\Sigma} f(\omega) \mu_{\omega}\left(h \circ \pi_{2} \circ \psi\right) d \mu_{0}(\omega)=(1.3) \int_{\Omega} f(\omega) \mu_{*, \omega}\left(h \circ \pi_{2}\right) d \mu_{0}(\omega) \\
= & \int_{\Omega} f(\omega) \alpha_{\omega}(h) d \mu_{0}(\omega) .
\end{aligned}
$$

Similarly,

$$
\int f(\omega) h \circ \pi_{2}(\omega, \zeta) d \mu_{*}^{\prime}(\omega, \zeta)=\int_{\Omega} f(\omega) \alpha_{\omega}^{\prime}(h) d \mu_{0}(\omega) .
$$

Recall $\mu_{*}=\mu_{*}^{\prime}$, and define

$$
S: L^{1}\left(\Omega, \mu_{0}\right) \rightarrow M\left(K^{\prime}\right): S(f) \cdot h=\int_{\Omega} f(\omega) \mu_{*, \omega}\left(h \circ \pi_{2}\right) d \mu_{0}(\omega) .
$$

The Dunford-Pettis theorem ([4], [9]) applies; there exists a unique 
(up to sets of $\mu_{0}$-measure zero) map $\sigma: \Omega \rightarrow M\left(K^{\prime}\right): \omega \rightarrow \sigma_{\omega}$ such that $S(f) \cdot h=\int_{\Omega} f(\omega) \sigma_{\omega}(h) d \mu_{0}(\omega)$ for all $f, h$. Hence $\alpha(\omega)=\sigma_{\omega}=\alpha^{\prime}(\omega)$ $\mu_{0}-$ a.e.; since Supp $\mu_{*, \omega}$ and Supp $\mu_{*, \omega}^{\prime}$ are subsets of $\{\omega\} \times K^{\prime}, \mu_{*, \omega}=$ $\mu_{*, \omega}^{\prime}$. The rest of the proof of 2.2 is the same as before.

THEOREM 2.4. 2.3 remains true if $K$ acts freely on $\Sigma$ (with, of course, $\Omega=\Sigma / K)$.

We say $K$ acts freely on $\Sigma$ if $(K, \Sigma)$ is a transformation group such that, if $\zeta \cdot \sigma=\sigma$ for some $\zeta \in K$ and $\sigma \in \Sigma$, then $\zeta=$ idy in $K$.

Proof. Using the technique of ([11], $\S 1)$, we construct a Borel isomorphism $\varphi$ of $\Omega \times K$ onto $\Sigma$ which (i) maps $\{\omega\} \times K$ homeomorphically onto $\pi^{-1}(\omega) \subset \Sigma$ for all $\omega \in \Omega(\pi: \Sigma \rightarrow \Omega$ is the quotient map); (ii) is $\eta$-proper for every $\eta \in M(\Omega \times K)$. If $t \in T$, define $t_{\varphi}: \Sigma \rightarrow \Sigma$ : $t_{\varphi}=\varphi \circ t \circ \varphi^{-1}$; one obtains a flow $\left(\Omega \times K, T_{\varphi}\right)$, where $T_{\varphi}$ consists of Borel measurable maps which are $\eta$-proper for every $\eta \in M(\Omega \times K)$. We may apply all the steps of 2.2 (with the modifications of 2.3) to the flow $\left(\Sigma \times K, T_{\varphi}\right)$. (In step (10), some extra work must be done because $T_{\varphi}$ does not consist of continuous maps, but the changes are straightforward.)

Notation 3.1. Let $B(t)$ be a minimal function (1.11), with $G(t)=\int_{0}^{t} B(s) d s$. Let $\Omega$ be the hull of $B$, and define $b \in C(\Omega)$ and $\omega_{0} \in \Omega$ so that $b\left(\omega_{0} \cdot t\right)=B(t)(t \in R)$. If $B$ is almost periodic (a.p.), let $\mu_{0}$ be normalized Haar measure on $\Omega$ (see 1.11). By uniqueness of Haar measure and $1.10, \mu_{0}$ is $R$-ergodic; it is the only ergodic measure on $\Omega$.

3.2. Consider the set of two-dimensional ordinary differential equations $E(\omega): \dot{x}=\left(\begin{array}{ll}0 & 0 \\ b(\omega \cdot t) & 0\end{array}\right) \times\left(x \in R^{2}, \omega \in \Omega\right)$. (We read $E(\omega)$ as "the equation corresponding to $\omega$ ".) The solutions to these ODEs generate a flow on $\Omega \times \boldsymbol{R}^{2}$, as follows: $(\bar{\omega}, \bar{x}) \cdot t=(\bar{\omega} \cdot t, x(t))$, where $x(t)$ is the solution to $E(\bar{\omega})$ with initial condition $x(0)=\bar{x}$. The flow $\left(\Omega \times \boldsymbol{R}^{2}, \boldsymbol{R}\right)$ is an example of a linear skew-product flow ([14], [15]). It is called "linear" because each $\operatorname{map} N_{t, \omega}:\{\omega\} \times \boldsymbol{R}^{2} \rightarrow\{\omega \cdot t\} \times \boldsymbol{R}^{2}$ : $(\omega, x) \rightarrow(\omega, x) \cdot t$ is linear. Let $P^{1}=$ projective one-space $=$ the set of lines through the origin in $\boldsymbol{R}^{2}$. By linearity, each map $N_{t, \omega}$ takes a line in $\{\omega\} \times \boldsymbol{R}^{2}$ to a line in $\{\omega \cdot t\} \times \boldsymbol{R}^{2}$; hence $\left(\Omega \times \boldsymbol{R}^{2}, \boldsymbol{R}\right)$ induces a flow $\left(\Omega \times P^{1}, R\right)$. We let $\Sigma=\Omega \times P^{1}, \pi: \Sigma \rightarrow \Omega:(\omega, \zeta) \rightarrow \omega$. Note $P^{1}$ is homeomorphic to a circle.

3.3. We can describe $(\Sigma, R)$ more usefully. Let $S^{1} \subset R^{2}$ be the 
unit circle, with polar coordinate $\theta$. We may visualize $P^{1}$ as that part of $S^{1}$ such that $-\pi / 2 \leqq \theta \leqq \pi / 2$, with $\theta=-\pi / 2$ and $\theta=\pi / 2$ identified. We will coordinatize $P^{1}$ with $\theta$ where $-\pi / 2<\theta \leqq \pi / 2$ (note the strict inequality). The flow $(\Sigma, \boldsymbol{R})$ may now be given as follows: (i) if $(\omega, \theta) \in \Sigma$ with $-\pi / 2<\theta<\pi / 2$, then $(\omega, \theta) \cdot t=(\omega \cdot t$, $\tan ^{-1}\left(\theta+\int_{0}^{t} b(\omega \cdot s) d s\right)$; (ii) if $\theta=\pi / 2$, then $(\omega, \pi / 2) \cdot t=(\omega, \pi / 2)(t \in \boldsymbol{R})$. One sees this by solving equations $E(\omega)$.

Definitions 3.4. Note that $\Sigma_{0}=\{(\omega, \pi / 2) \mid \omega \in \Omega\}$ is a compact invariant subset of $\Sigma$. The projection $\pi: \Sigma \rightarrow \Omega$ induces a homeomorphism $\pi_{0}=\left.\pi\right|_{\Sigma_{0}}$ of $\Sigma_{0}$ onto $\Omega$ which commutes with the flows. If $\mu_{0}$ is an ergodic measure on $\Sigma$, then $\eta=\pi_{0}^{-1}\left(\mu_{0}\right)$ is a measure on $\Sigma_{0}$. If we view $\eta$ as a measure on $\Sigma$ in the obvious way, then $\eta$ is supported on $\Sigma_{0}$ and ergodic with respect to $(\Sigma, \boldsymbol{R})$.

LeMMA 3.5. Let $\Omega, \Sigma, \Sigma_{0}, \pi_{0}$ be as above (except that, in this lemma, $\Omega$ need not be metric). Suppose that every measure on $\Sigma$ which is ergodic with respect to $(\Sigma, \boldsymbol{R})$ has the form $\pi_{0}^{-1}\left(\mu_{0}\right)$ for some ergodic $\mu_{0}$ on $\Omega$. Let $f \in C(\Sigma)$ satisfy $\left.f\right|_{\Sigma_{0}}=0$. Let $\nu_{t, \sigma}(g)=$ $1 / t \int_{0}^{t} g(\sigma \cdot s) d s(\sigma \in \Sigma, t \in \boldsymbol{R}, g \in C(\Sigma))$. Then, given $\varepsilon>0, \exists T$ such that $|t| \geqq T \Longrightarrow\left|\nu_{t, o}(f)\right|<\varepsilon$.

Proof. Observe that $\eta(f)=0$ for every ergodic $\eta$ on $\Sigma$. Suppose for contradiction that $f$ does not satisfy the conclusion of 3.5. Let $t_{n}$ and $\omega_{n}$ be points such that $\left|t_{n}\right|>n$ and $\left|\nu_{t_{n}, \omega_{n}}(f)\right| \geqq \varepsilon$. Choose a subnet $\left(t_{\alpha}, \sigma_{\alpha}\right)$ of $\left(t_{n}, \omega_{n}\right)$ such that $\nu_{t_{\alpha}, \omega_{\alpha}}$ converges to some $\nu \in$ $M_{1}(\Sigma)$. Then $\nu(f) \neq 0$. We may assume $t_{\alpha} \rightarrow+\infty, \omega_{\alpha} \rightarrow \omega$. But these two conditions imply that $\nu$ is invariant. Now, it is well-known that the set of invariant measures is the closed convex hull of the set of ergodic measures (in the topology of pointwise convergence). Hence $\nu(f)=0$. This contradiction proves 3.5.

Definition 3.6. If $I \subset \boldsymbol{R}, b \in C(\Omega)$, and $\omega \in \Omega$, let $A(n, I, \omega, b)=$ $1 / 2 n \gamma\left\{t \in[-n, n] \mid g_{\omega}(t) \in I\right\}$, where $g_{\omega}(t)=\int_{0}^{t} b(\omega \cdot s) d s$ and $\gamma$ is Lebesgue measure on $\boldsymbol{R}(\gamma[0,1]=1)$. When confusion cannot arise, we will write $A(n, I, \omega)$.

Proposition 3.7. Assume there is a compact set $I \subset \boldsymbol{R}$ such that $\overline{\lim }_{n \rightarrow \infty} A\left(n, I, \omega_{0}\right)>0$ for some $\omega_{0} \in \Omega$. Then there is an ergodic $\mu_{0}$ on $\Omega$ and at least two ergodic measures $\eta, \mu$ on $\Sigma$ such that $\pi(\eta)=\mu_{0}$.

Proof. There is at least one ergodic measure on $\Omega([13])$. If 
the conclusion of 3.7 is false, then, given an ergodic $\mu_{0}$ on $\Omega$, the measure $\eta=\pi_{0}^{-1}\left(\mu_{0}\right)$ (see 3.4 ) is the only ergodic measure on $\Sigma$ satisfying $\pi(\eta)=\mu_{0}$. Let $I_{1}=\tan ^{-1} I \subset(-\pi / 2, \pi / 2) \subset P^{1}$, and let $\Sigma_{1}=$ $\Sigma \times I_{1}$. Let $f$ be a continuous, nonnegative function which is 0 on $\Sigma_{0}$ and 1 on $\Sigma_{1}$. Let $E_{n}=\left\{t \in[-n, n] \mid g_{\omega_{0}}(t) \in I\right\}$. Then $f\left(\left(\omega_{0}, 0\right) \cdot t\right)=$ 1 if $t \in E_{n}$. Hence $\overline{\lim }_{n \rightarrow \infty} 1 / 2 n\left[\int_{0}^{n} f\left(\omega_{0}, 0\right) \cdot s d s+\int_{-n}^{0} f\left(\omega_{0}, 0\right) \cdot s d s\right]>0$. This contradicts $3.5 ; 3.7$ is proved.

Let $\mu_{0}$ be an ergodic measure on $\Omega$ which satisfies the condition of 3.7. If $B(t)$ is a.p., then $\mu_{0}$ is normalized Haar measure (3.1).

THEOREM 3.8. (a) Suppose there exist $\omega_{0} \in \Omega$ and a compact $I \subset \boldsymbol{R}$ such that $\varlimsup_{n \rightarrow \infty} A\left(n, I, \omega_{0}\right)>0$. Then there is a $\mu_{0}$-measurable function $r$ on $\Omega$ such that $r(\omega \cdot t)-r(\omega)=\int_{0}^{t} b(\omega \cdot s) d s \mu_{0}$-a.e. for each $t \in \boldsymbol{R}$.

(b) If $B(t)$ is a.p., then $r$ may be chosen so that $r(\omega \cdot t)-r(\omega)=$ $\int_{0}^{t} b(\omega \cdot s) d s$ for all $\omega \in \Omega, t \in \boldsymbol{R}$.

Proof. (a) Using 3.6, we can find an ergodic $\mu_{0}$ on $\Omega$ and ergodic measures $\eta=\pi_{0}^{-1}\left(\mu_{0}\right)$ and $\mu \neq \eta$ on $\Sigma$ such that $\pi(\eta)=\pi(\mu)=\mu_{0}$. Let $\lambda: \Omega \rightarrow M_{1}(\Sigma): \omega \rightarrow \lambda_{\omega}$ be a disintegration of $\mu$ with respect to $\mu_{0}(1.6)$. Using uniqueness in $1.6(1.6(\mathrm{~b}))$, it is easy to see that $(*) \lambda_{\omega \cdot t}=\left(\lambda_{\omega}\right) \cdot t \mu_{0}-$ a.e. for each $t \in \boldsymbol{R}$.

By 2.2, there exists an integer $n$ such that $\left|\operatorname{Supp} \lambda_{\omega}\right|=n$ on a set $B \subset \Omega$ of $\mu_{0}$-measure 1. For $\omega \in B$, we write $\lambda_{\omega}=\sum_{i=1}^{n} \alpha_{i}(\omega) \delta_{\left(\omega, \theta_{i}(\omega)\right)}$

$$
\left(\delta=\text { Dirac measure), where } \theta_{0}(\omega)<\theta_{2}(\omega)<\cdots<\theta_{n}(\omega) .\right.
$$

Let $B_{1}=\left\{\omega \in B \mid \theta_{n}(\omega)=\pi / 2\right\}$ (recall $\theta$ has range $\pi / 2<\theta \leqq \pi / 2$ ).

By $\left({ }^{*}\right)$ and invariance of $\Sigma_{0}, B_{1}$ is $R$-invariant in the sense of 2.10 .

We claim $B_{1}$ is $\mu_{0}$-measurable. Let $\Gamma \subset B$ be a compact set such that $\left.\lambda\right|_{\Gamma}$ is continuous. It suffices to show that $B_{1} \cap \Gamma$ is closed. Let $\omega_{l} \in B_{1} \cap \Gamma, \omega_{l} \rightarrow \omega \in B$. Choosing a subsequence, we may assume that $\alpha_{i}\left(\omega_{l}\right) \rightarrow \bar{\alpha}_{i}, \theta_{i}\left(\omega_{l}\right) \rightarrow \bar{\theta}_{i}$. It is easy to see that $\left\{\theta_{1}(\omega), \cdots, \theta_{n}(\omega)\right\} \subset$ $\left\{\bar{\theta}_{i} \mid \bar{\alpha}_{i} \neq 0(1 \leqq i \leqq n)\right\}$. Hence the two sets are equal, and no $\bar{\alpha}_{i}$ can be zero. Since $\bar{\theta}=\pi / 2$, we must have $\theta_{n}(\omega)=\pi / 2$. So $B_{1}$ is $\mu_{0}$ measurable.

Since $\mu_{0}$ is ergodic, $\mu_{0}\left(B_{1}\right)=0$ or 1 . It cannot be 1 . For, suppose it is. The ergodic measures $\eta$ and $\mu$ are mutually singular (considerably more is true; see, e.g., [13], pp. 496-508). Let $D_{1}$ and $D_{2}$ be Borel sets in $\Sigma$ such that $1=\eta\left(D_{1}\right)=\mu\left(D_{2}\right), D_{1} \cap D_{2}=\phi$. Clearly $\eta\left(D_{1} \cap \Sigma_{0}\right)=1$. This implies that, for $\mu_{0}-$ a.a. $\omega$, one has $D_{1} \cap \Sigma_{0} \cap$ $\pi^{-1}(\omega)=\phi$. Clearly $\eta\left(D_{1} \cap \Sigma_{0}\right)=1$. This implies that, for $\mu_{0}-$ a.a. $\omega$, 
one has $D_{2} \cap \Sigma_{0} \cap \pi^{-1}(\omega)=\dot{\phi}$. Since $\mu_{0}\left(B_{1}\right)=1$, we have $\lambda_{\omega}\left(D_{2}\right)<1$ for $\mu_{0}$ - a.a. $\omega$. But $1=\mu\left(D_{2}\right)=(1.6(\mathrm{c}))$

$$
\int_{\Omega} \lambda_{\omega}\left(D_{2}\right) d \mu_{0}(\omega)<1
$$

The contradiction shows that $\mu_{0}\left(B_{1}\right)=0$.

Let $B_{2}=\Omega \sim B_{1}$; then $\mu_{0}\left(B_{2}\right)=1$. Let $D_{3}=\left\{(\omega, \theta) \in \Sigma \mid \omega \in B_{2}\right.$, $\left.\theta=\max _{1 \leqq i \leqq n} \theta_{i}(\omega)\right\}$. Then $D_{3}$ is $R$-invariant $\left(\left(^{*}\right)\right.$ and the fact that the flow on $\Sigma$ preserves the $\theta$-order). We claim $D_{3}$ is $\mu$-measurable. Let $\Gamma \subset B_{2}$ be a compact set such that $\left.\lambda\right|_{\Gamma}$ is continuous, and let $\Gamma_{1}=\pi^{-1}(\Gamma)$. We show that $D_{3} \cap \Gamma_{1}$ is closed. Let $\left(\omega_{l}, \theta_{l}\right) \in D_{3} \cap \Gamma_{1}$, with $\left(\omega_{l}, \theta_{l}\right) \rightarrow(\omega, \theta)$. Then $\omega \in B_{2}$, and $\lambda_{\omega_{l}} \rightarrow \lambda_{\omega}$. Choosing a subsequence, we assume $\alpha_{i}\left(\omega_{l}\right) \rightarrow \bar{\alpha}_{i}, \theta_{i}\left(\omega_{l}\right) \rightarrow \bar{\theta}_{i}$. Now each $\theta_{l}$ is equal to $\theta_{n}\left(\omega_{l}\right)$. Hence $\theta=\bar{\theta}_{n}$. As before, $\left\{\theta_{1}(\omega), \cdots, \theta_{n}(\omega)\right\}=\left\{\bar{\theta}_{1}, \cdots, \bar{\theta}_{n}\right\}=$ $\left\{\bar{\theta}_{1}, \cdots, \theta\right\}$. We claim that $\theta=\theta_{n}(\omega)$. Since the $\theta_{i}\left(\omega_{l}\right)$ were arranged in increasing order, it suffices to show that $\bar{\theta}_{1} \neq-\pi / 2$. But, if this were not the case, then $\theta_{n}(\omega)$ would be $\pi / 2$. Since it is not $\left(\omega \in B_{2}\right)$, we have $\theta=\theta_{n}(\omega)$, and hence $(\omega, \theta) \in D_{3}$.

Either $\mu\left(D_{3}\right)=0$ or $\mu\left(D_{3}\right)=1$. But $\mu\left(D_{3}\right)=\int_{\Omega} \lambda_{\omega}\left(D_{3}\right) d \mu_{0}(\omega)$, and $\lambda_{\omega}\left(D_{3}\right)>0$ on $B_{2}$. Hence $\mu\left(D_{3}\right)=1$. This implies $\lambda_{\omega}$ is supported on the point $(\omega, \theta)$ if $\omega \in B_{2}$; i.e., $\lambda_{\omega}=\delta_{(\omega, \theta)}$.

We now define $r$. If $\omega \in B_{2}$, let $(\omega, \theta)$ be the corresponding point in $D_{3}$, and let $r(\omega)=\tan \theta$. If $\omega \notin B_{2}$, define $r$ arbitrarily. Since $D_{3}$ is $R$-invariant, one has $r(\omega \cdot t)=\tan \tan ^{-1}\left(\theta+\int_{0}^{t} b(\omega \cdot s) d s=\right.$ $r(\omega)+\int_{0}^{t} b(\omega \cdot s) d s \mu_{0}$ - a.e. for each $t \in \boldsymbol{R}$. Also, it follows immediately from the proof of $\mu$-measurability of $D_{3}$ that is $\mu_{0}$-measurable. This completes the proof of (a).

(b) Let $\lambda_{\omega}$ be the disintegration of $\mu$ with respect to $\mu_{0}$ of (a). We first arrange that $\lambda_{\omega \cdot t}=\left(\lambda_{\omega}\right) \cdot t$ for all $\omega \in \Omega$ and $t \in R$. To do this, let $\rho$ be a strong lifting of $M^{\infty}\left(\Omega, \mu_{0}\right)$ commuting with translations (1.8). As in ([9], Chpt. VI, Prop. 1), we may define a new disintegration $\lambda^{\prime}$ of $\mu$ with respect to $\mu_{0}$ by the formula $\lambda_{\omega}^{\prime}(f)=$ $\rho(g)(\omega)$, where $g: \Omega \rightarrow \boldsymbol{R}: g(\overline{\boldsymbol{\omega}})=\lambda_{\bar{\omega}}(f)(f \in C(\Sigma))$. It is easily seen that $\lambda_{\omega \cdot t}^{\prime}=\left(\lambda_{\omega}^{\prime}\right) \cdot t$ for all $\omega, t$.

Now go through the proof of (a) with $\lambda^{\prime}$ in place of $\lambda$. One finds that $B_{2}$ is strictly $R$-invariant (in the sense of 1.10). If $\omega \in B_{2}$, define $r(\omega)=\tan \theta$; define $r$ on $\Omega \sim B_{2}$ in any manner so that $r(\omega \cdot t)-$ $r(\omega)=\int_{0}^{t} b(\omega \cdot s) d s$ holds. Then this equation holds for all $\omega, t$. As in (a), $r$ is $\mu_{0}$-measurable. The proof of (b) is complete.

By restating the hypotheses of 3.8, we obtain a theorem whose converse is also true. 
THEOREM 3.9. The following are equivalent.

(a) There is an ergodic measure $\mu_{0}$ on $\Omega$, a set $\Omega_{1} \subset \Omega$ with $\mu_{0}\left(\Omega_{1}\right)=1$ and compact sets $I_{\omega} \subset R$ such that $\lim _{n \rightarrow \infty} A\left(n, I_{\omega}, \omega\right)$ exists and is positive $\left(\omega \in \Omega_{1}\right)$.

(b) There is an ergodic measure $\mu_{0}$ on $\Omega$ and a $\mu_{0}$-measurable function $r$ such that $r(\omega \cdot t)-r(\omega)=\int_{0}^{t} b(\omega \cdot s) d s \mu_{0}$-a.e. for each $t \in$ $\boldsymbol{R}$. If $\boldsymbol{B}(t)$ is a.p., then $r$ may be chosen so that equality holds for all $\omega, t$.

Proof. $\quad(a) \Rightarrow(b)$ : follows from 3.8 .

(b) $\Rightarrow\left(\right.$ a): Let $J$ be any compact set such that $B=r^{-1}(J)$ has positive $\mu_{0}$-measure. Let $\varphi_{B}$ be the characteristic function of $B$. By the Birkhoff ergodic theorem ([13]), $1 / t \int_{0}^{t} \varphi_{B}(\omega \cdot s) d s \rightarrow \mu_{0}(B)$ as $t \rightarrow \infty$ and as $t \rightarrow-\infty$, for $\mu_{0}-$ a.a. $\omega$. Fix such an $\omega$. Note that $\omega \cdot s \in B$ iff $r(\omega \cdot s) \in J$. Let $I_{\omega}=\{s-r(\omega) \mid s \in J\}$. Then $\lim _{n \rightarrow \infty} A\left(n, I_{\omega}, \omega\right)=$ $\mu_{0}(B)>0$.

REMARKS 3.10. (a) Since (b) $\Rightarrow$ (a) in 3.9 , we can conclude that the hypothesis of 3.8 implies 3.9 (a). Thus the relative density hypothesis "extends from a point to almost all of the hull".

(b) Since $J$ can be chosen to be an interval of arbitrarily small length, so can the sets $I_{\omega}$.

(c) Theorems 3.8 and 3.9 say nothing about $\mu_{0}$-integrability of $r$.

(d) Using the techniques of $\S 3$, one can prove results analogous to $3.8,3.9$ for minimal integer flows $(\Omega, T)$ ( $\Omega$ a compact metric space, $T: \Omega \rightarrow \Omega$ a homeomorphism). Let $b: \Omega \rightarrow \boldsymbol{R}$ be continuous. The analogues of $3.8,3.9$ are obtained by simply replacing $\int_{0}^{t} b(\omega \cdot s) d s$ by $g_{\omega}(m)=\sum_{k=0}^{m} b\left(\omega \cdot T^{k}\right)$ throughout (if $m$ is negative, let $g_{\omega}(m)=$ $\left.\sum_{k=0}^{-m} b\left(\omega \cdot T^{-k}\right)\right)$, and by replacing $A(n, I, \omega, b)$ by $1 / 2 n$ card $\{m \in[-n$, $\left.n] \mid g_{\omega}(m) \in I\right\}$.

(e) Let $(\Omega, R)$ be a.p. minimal. Let $C_{0}(\Omega)=\{b \in C(\Omega) \mid b$ has mean value zero\}. There is a $b_{0} \in C_{0}(\Omega)$, and a $\mu_{0}$-measurable, discontinuous function $r_{0}: \Omega \rightarrow \boldsymbol{R}$ such that

$$
r_{0}(\omega t)-r_{0}(\omega)=\int_{0}^{t} b_{0}(\omega \cdot s) d s(\omega \cdot \Omega, t \in \boldsymbol{R}) .
$$

One can prove this by constructing $r_{0}$, using a method similar to that of ([7], p. 585). See also ([10]). We will not give details here. Now, in 4.3 below, it is shown that $V=\left\{v \in C_{0}(\Omega) \mid \int_{0}^{t} v(\omega \cdot s) d s\right.$ is bounded $(\omega \in \Omega)\}$ is dense in $C_{0}(\Omega)$. Then $b_{0}+V$ is also dense in $C_{0}(\Omega)$. Hence the set of functions $b \in C_{0}(\Omega)$ with a $\alpha$ " $\mu_{0}$-measurable, 
discontinuous antiderivative" $r$, is dense in $C_{0}(\Omega)$.

4. In this section, we show that "most" a.p. functions satisfy neither conditions of 3.9. To make this precise, we alter our point of view somewhat, and consider some almost periodic minimal set $([5])(\Omega, R)$. If $\Omega$ is metrizable, then $\Omega$ is the hull of some a.p. function $B(t)$. However, we will not assume $\Omega$ is metrizable. The result is then the following. Suppose $(\Omega, \boldsymbol{R})$ is not a periodic flow (i.e., $\Omega$ is not the hull of a periodic function), and let $C_{0}(\Omega)=\{b \in$ $C(\Omega) \mid b$ has mean value zero ; then there is a residual subset $C_{1}$ of $C_{0}(\Omega)$ such that $b \in C_{1} \Rightarrow \lim _{n \rightarrow \infty} 1 / 2 n \gamma\left\{t \in[-n, n] \mid \int_{0}^{t} b(\omega \cdot s) d s \in I\right\}=0$ for all $\omega \in \Omega$ and all compact $I \subset \boldsymbol{R}$.

NATAtion 4.1. Let $(\Omega, \boldsymbol{R})$ be an a.p. minimal set. As in 3.6, let $A(n, I, \omega, b)=1 / 2 n \gamma\left\{t \in[-n, n] \mid \int_{0}^{t} b(\omega \cdot s) d s \in I\right\}$ for $b \in C(\Omega)$ and compact $I \subset \boldsymbol{R}$. Recall that the mean value of $b \in C(\Omega)$ equals

$$
\int_{\Omega} b(\omega) d \mu_{0}(\omega)\left(\mu_{0}=\text { normalized Haar measure on } \Omega\right) \text {. }
$$

Let $C_{0}(\Omega)=\{b \in C(\Omega) \mid b$ has mean value zero $\}$. Give $C(\Omega)$ the supnorm topology.

LemMA 4.2. Suppose $(\Omega, \boldsymbol{R})$ is not a periodic flow. Let $0<\varepsilon<1$ and compact $I \subset \boldsymbol{R}$ be given. Then there is a $c \in C_{0}(\Omega)$ with $\|c\|=1$ such that $A(n, I, \omega, c)<\varepsilon$ for all $\omega$ if $n$ is sufficiently large.

Proof. First pick $\omega_{0} \in \Omega$ and $b \in C_{0}(\Omega)$. We may assume that $B(t)=b\left(\omega_{0} \cdot t\right)$ is not periodic in $t$. Expand $B(t)$ in a Bohr-Fourier series: $B(t)=\sum_{k=1}^{\infty} a_{k} e^{i \lambda_{k} t}$. We may assume $\lambda_{k} \neq 0$ for all $k$. Either (i) $\lambda_{m} / \lambda_{l}$ is rational for all $m$ and $l$, in which case $\underline{\lim }\left|\lambda_{m}\right|=0$, or (ii) $\lambda_{m} / \lambda_{l}$ is irrational for some $m, l$. Let $\Omega_{1}$ be the hull of $B(t)$, and write $B_{\tau}(t)=B(t+\tau)(t, \tau \in \boldsymbol{R})$. The correspondence $\omega_{0} \cdot \tau \rightarrow B_{\tau}$ : $\left\{\omega_{0} \cdot t \mid t \in R\right\} \rightarrow \Omega_{1}$ is uniformly continuous, hence extends to a surjection $\tau_{1}: \Omega \rightarrow \Omega_{1}$ which commutes with the flows.

Next, let $K$ be the unit circle, and let $K_{\infty}=\prod_{k=1}^{\infty} K$. Define a flow $\left(K_{\infty}, R\right)$ as follows: $\left(e^{i \theta_{k}}\right)_{k=1}^{\infty} \cdot t=\left(e^{i\left(\theta_{k}+\lambda_{k} t\right)}\right)_{k=1}^{\infty}$. The correspondence $B_{\tau} \rightarrow\left(e^{i \lambda_{k} t}\right)_{k=1}^{\infty}:\left\{B_{\tau} \mid \tau \in \boldsymbol{R}\right\} \rightarrow K_{\infty}$ is uniformly continuous, hence extends to a continuous map $\tau_{2}: \Omega_{1} \rightarrow K_{\infty}$. Let $\Omega_{2}=$ Image $\left(\tau_{2}\right)$; then $\Omega_{2}$ is compact invariant, and $\tau_{2}: \Omega_{1} \rightarrow K_{\infty}$ commutes with the flows.

Now consider case (i). Define $c_{m}: K_{\infty} \rightarrow \boldsymbol{R}:\left(e^{i \theta_{k}}\right)_{k=1}^{\infty} \rightarrow \cos \theta_{m}$. Note

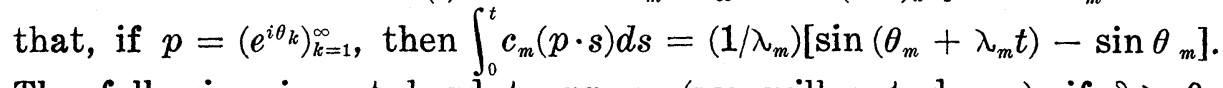
The following is not hard to prove (we will not do so): if $\delta>0$, 
and if $f_{j}(x, \delta)=(1 / 2 j) \gamma\{y \in[-j, j]|| \sin (x+y)-\sin x \mid \leqq \delta\}(0<j \in \boldsymbol{R}$, $x \in \boldsymbol{R})$, then given $\varepsilon>0, \exists \delta>0$ and $J$ such that $j \geqq J \Rightarrow f_{j}(x, \delta) \leqq \varepsilon$ uniformly in $x$. Let $I$ and $\varepsilon$ be as in the statement of 4.2. Choose $M>0$ so that $I \subset[-M, M]$. Choose $\delta>0$ and $J$ so that $j \geqq J \Longrightarrow$ $f_{j}(x, \delta)<\varepsilon$ for all $x \in \boldsymbol{R}$. There is a $\lambda_{m}$ such that $\left|\lambda_{m}\right| \cdot M<\delta$. Let $n=j /\left|\lambda_{m}\right|$. Note that $(1 / 2 n) \gamma\left\{t \in[-n, n] \mid \int_{0}^{t} c_{m}(p \cdot s) d s \in[-M, M]\right\}=$ $(1 / 2 j) \gamma\left\{\tau \in[-j, j]|| \sin \left(\theta_{m}+\tau\right)-\sin \theta_{m} \mid \leqq \delta\right\}<\varepsilon$ if $j \geqq J$, for all $p \in$ $K_{\infty}$. Let $C=c_{m} \circ \tau_{2} \circ \tau_{1}$, and choose $N \geqq J /\left|\lambda_{m}\right|$. Then $n \in N \Rightarrow A(n$, $I, \omega, c)<\varepsilon$ for all $\omega \in \Omega$.

Finally, consider case (ii). Suppose $\lambda_{m} / \lambda_{l}$ is irrational. The $\operatorname{map}\left(e^{i \lambda_{k} t}\right)_{k=1}^{\infty} \rightarrow\left(e^{i \lambda_{m} t}, e^{i \lambda_{l} t}\right)$ of $\left\{\left(e^{i \lambda_{k} t}\right)_{k=1}^{\infty} \mid t \in \boldsymbol{R}\right\}$ into the 2-torus $K \times K=$ $K^{2}$ is uniformly continuous, hence extends to a continuous map $\tau_{3}$ of $\Omega_{2}$ onto $K^{2}$ which commutes with the flows (the flow on $K^{2}$ is of course the irrational twist defined by $\lambda_{m}$ and $\left.\lambda_{l}\right)$. For integers $\tau$ and $s$, define $C_{\tau s}: K^{2} \rightarrow \boldsymbol{R}:\left(e^{i \theta}, e^{i \varphi}\right) \rightarrow \cos (\tau \theta+s \varphi)$. We can choose $\tau$ and $s$ so that $\left|\tau \lambda_{m}+s \lambda_{l}\right|$ is as small as we please. Therefore, we can apply an argument like that used in case (i) to show that, if $C=C_{\tau s} \circ \tau_{3} \circ \tau_{2} \circ \tau_{1}$, then (for appropriate $\tau$ and $s$ ) $C$ satisfies 4.2.

LEMmA 4.3. Let $V=\left\{b \in C_{0}(\Omega) \mid \int_{0}^{t} b(\omega \cdot s) d s\right.$ is uniformly bounded $(\omega \in \Omega, t \in \boldsymbol{R})\}$. Then $V$ is dense in $C_{0}(\Omega)$.

Proof. Let $\widetilde{b} \in C_{0}(\Omega), \omega_{0} \in \Omega, \widetilde{B}(t)=\widetilde{b}\left(\omega_{0} \cdot t\right), \widetilde{B}(t)=\sum_{k=0}^{\infty} a_{k} e^{i \lambda_{k} t}$.

Then $\widetilde{B}(t)$ may be uniformly approximated by trigonometric polynomials without constant term whose frequencies are among the $\lambda_{k} s([6])$. Such a polynomial defines a function $b$ on $\Omega$ such that $\int_{0}^{t} b(\omega \cdot s) d s$ is uniformly bounded as a function of $\omega \in \Omega$ and $t \in \boldsymbol{R}$. The lemma follows.

THEOREM 4.4. Let $(\Omega, \boldsymbol{R})$ be a nonperiodic, almost periodic minimal set. Then there is a residual subset $C_{1}$ of $C_{0}(\Omega)$ such that $\lim _{n \rightarrow \infty} A(n, I, \omega, b)=0$ for all $\omega \in \Omega$ and all compact $I \subset \boldsymbol{R}\left(b \in C_{1}\right)$.

Proof. Let $Q(I, k, N)=\left\{b \in C_{0}(\Omega) \mid\right.$ for some $\omega \in \Omega$ (depending on $b$ ), one has $A(n, I, \omega, b) \geqq 1 / k$ for $n \geqq N\}$. By 3.8 and 3.3, $\bigcup_{I \subset R}$ $\bigcup_{k=1}^{\infty} \bigcup_{N=1}^{\infty} Q(I, k, N)=\left\{b \in C_{0}(\Omega) \mid\right.$ for some $\omega \in \Omega$ and some compact $\left.I \subset \boldsymbol{R}, \overline{\lim }_{n \rightarrow \infty} A(n, I, \omega, b)>0\right\}$.

Without loss of generality, we can restrict attention to sets $I$ of the form $[-\alpha, \alpha]$, where $\alpha$ is an integer. It is easily seen that, if $I=[-\alpha, \alpha]$, then $\operatorname{cls} Q(I, k, N) \subset Q\left(I_{1}, k, N\right)$, where $I_{1}=[-\alpha-1$, $\alpha+1]$. Hence, if 4.4 is false, then some $Q(I, k, N)$ contains a ball 
$W$ of radius $\delta>0$. By 4.3, we may suppose that, if $a$ is the center of $W$, then $\int_{0}^{t} a(\omega \cdot s) d s$ is in some compact interval $I_{2}$ for all $t, \omega$. Let $I \cup I_{2} \subset\left[-\alpha_{1}, \alpha_{1}\right]$, then let $I_{3}=\left[-2 \alpha_{1} / \delta, 2 \alpha_{1} / \delta\right]$. Apply $4.2 \mathrm{with}$ $I_{3}$ replacing $I$ and $1 / k$ replacing $\varepsilon$. We obtain a function $c$ such that $a+\delta c \in W$ and $A(n, I, \omega, a+\delta c)<1 / k$ for all $\omega$ if $n$ is sufficiently large. We have arrived at a contradiction, and proved 4.4.

REMARKS 4.5. (a) By 4.4 and 3.9, residually many $b \in C_{0}(\Omega)$ admit no $\mu_{0}$-measurable $r$ with $r(\omega \cdot t)-r(\omega)=\int_{0}^{t} b(\omega \cdot s) d s$.

(b) A theorem analogous to 4.4 holds for integer a.p. minimal flows $(\Omega, T)$. The statement of this theorem is obtained (as in $3.10(\mathrm{~d}))$ by simply replacing $\int_{0}^{t} b(\omega \cdot s) d s$ with $g_{\omega}(m)=\sum_{k=0}^{m} b\left(\omega \cdot T^{k}\right)$ $\left(\sum_{k=0}^{-n} b\left(\omega \cdot T^{-k}\right)\right.$ if $\left.n<0\right)$, and replacing $A(n, I, \omega, b)$ by $1 / 2 n$ card $\{m \in$ $\left.[-n, n] \mid g_{\omega}(m) \in I\right\}$. We must assume $(\Omega, T)$ is not periodic; i.e., that $T^{j}=\mathrm{idy}$ on $\Omega$ for no $j$.

\section{REFERENCES}

1. N. Bourbaki, Integration, Chpts. I-IV, 2nd ed., Hermann, Paris, 1965.

2. - Integration, Chpt. V, 2nd ed., Hermann, Paris, 1967.

3. - Integration, Chpt. VI, 1st ed., Hermann, Paris, 1959.

4. N. Dunford and J. B. Pettis, Linear operations on summable functions, Trans. Amer. Math. Soc., 47 (1940), 323-392.

5. R. Ellis, Lectures on Topological Dynamics, Benjamin, New York, 1969.

6. A. M. Fink, Almost Periodic Differential Equations, Lecture Notes in Mathematics \# 377, Springer-Verlag, New York, 1974.

7. H. Furstenberg, Strict ergodicity and transformations of the torus, Amer. J. Math., 83 (1961), 573-601.

8. A. and C. Ionescu-Tulcea, On the existence... locally compact group, Proc. Fifth Berk. Symp. Math. Stat. and Prob., 1965, V. 2, pt. 1, 63-97.

9. - Topics in the Theory of Lifting, Springer-Verlag, New York, 1969.

10. R. Johnson, $A$ measurable, non-continuous function on the 2-torus, preprint.

11. —, Disintegration of measures on compact transformation groups, to appear in Trans. Amer. Math. Soc.

12. J. L. Kelley, General Topology, D. Van Nostrand Co., Princeton, 1955.

13. V. V. Nemyckii and V. V. Stepanov, Qualitative Theory of Differential Equations, English trans., Princeton Math. series, \#22, Princeton Univ. Press, Princeton, 1960.

14. R. Sacker and G. Sell, Existence of dichotomies and invariant splittings for linear differential systems I, J. Differential Equations, 15 (1974), 429-458.

15. G. Sell, Linear Differential Systems, Lecture Notes, University of Minnesota, 1975.

Received February 15, 1978 and in revised form March 28, 1979.

University of Southern CaLiforNia

LOS ANGELES, CA 90007 
.././. ./FrontMatter/paper .pdf 


\section{Pacific Journal of Mathematics \\ Vol. 87, No. $2 \quad$ February, 1980}

Theagenis Abatzoglou, Unique best approximation from a $C^{2}$-manifold in Hilbert space ................................. 233

Gerald Arthur Anderson, $\Lambda$-homology cobordism bundles............. 245

Eric Bedford, Holomorphic mapping of products of annuli in $\mathbf{C}^{n} \ldots \ldots \ldots 271$

Gunnar Carlsson, On the stable splitting of $b o \wedge b o$ and torsion operations in connective $K$-theory .......................... 283

Lester Eli Dubins and David Samuel McIntyre Margolies, Naturally integrable functions ................................. 299

Leo Egghe, The Radon-Nikodým property, $\sigma$-dentability and martingales in locally convex spaces ............................. 313

Irving Leonard Glicksberg, Maps preserving translates of a function ..... 323

Hugh M. Hilden and Robert D. Little, Cobordism of branched covering

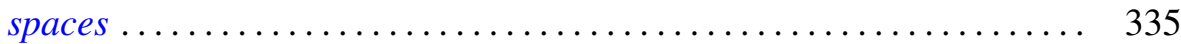

Russell Allan Johnson, Almost-periodic functions with unbounded integral .......................................... 347

Bruce Stephen Lund, The endomorphisms of a Dirichlet algebra ........ 363

John Henry McCleary, Mod $p$ decompositions of H-spaces; another approach........................................ 373

Arlan Bruce Ramsay, Subobjects of virtual groups ................ 389

Thomas R. Savage, Generalized inverses in regular rings ............ 455 Jaak Vilms, On curvature operators of bounded rank ......... 